Estimation of electrode location in a rat motor cortex by laminar analysis of electrophysiology and intracortical electrical stimulation

This article has been downloaded from IOPscience. Please scroll down to see the full text article.

2011 J. Neural Eng. 8046018

(http://iopscience.iop.org/1741-2552/8/4/046018)

View the table of contents for this issue, or go to the journal homepage for more

Download details:

IP Address: 141.211.173.82

The article was downloaded on 06/04/2012 at 16:25

Please note that terms and conditions apply. 


\title{
Estimation of electrode location in a rat motor cortex by laminar analysis of electrophysiology and intracortical electrical stimulation
}

\author{
A Yazdan-Shahmorad ${ }^{1}$, M J Lehmkuhle ${ }^{2}$, G J Gage ${ }^{1}$, T C Marzullo $^{1}$, \\ H Parikh ${ }^{1}$, R M Miriani ${ }^{1}$ and D R Kipke ${ }^{1}$ \\ ${ }^{1}$ Biomedical Engineering Department, University of Michigan, Ann Arbor, MI, USA \\ ${ }^{2}$ Department of Physiology, School of Medicine, University of Utah, UT, USA \\ E-mail: azadehy@umich.edu
}

Received 1 April 2011

Accepted for publication 27 May 2011

Published 20 June 2011

Online at stacks.iop.org/JNE/8/046018

\begin{abstract}
While the development of microelectrode arrays has enabled access to disparate regions of a cortex for neurorehabilitation, neuroprosthetic and basic neuroscience research, accurate interpretation of the signals and manipulation of the cortical neurons depend upon the anatomical placement of the electrode arrays in a layered cortex. Toward this end, this report compares two in vivo methods for identifying the placement of electrodes in a linear array spaced $100 \mu \mathrm{m}$ apart based on in situ laminar analysis of (1) ketamine-xylazine-induced field potential oscillations in a rat motor cortex and (2) an intracortical electrical stimulation-induced movement threshold. The first method is based on finding the polarity reversal in laminar oscillations which is reported to appear at the transition between layers IV and V in laminar 'high voltage spindles' of the rat cortical column. Analysis of histological images in our dataset indicates that polarity reversal is detected $150.1 \pm 104.2 \mu \mathrm{m}$ below the start of layer $\mathrm{V}$. The second method compares the intracortical microstimulation currents that elicit a physical movement for anodic versus cathodic stimulation. It is based on the hypothesis that neural elements perpendicular to the electrode surface are preferentially excited by anodic stimulation while cathodic stimulation excites those with a direction component parallel to its surface. With this method, we expect to see a change in the stimulation currents that elicits a movement at the beginning of layer $\mathrm{V}$ when comparing anodic versus cathodic stimulation as the upper cortical layers contain neuronal structures that are primarily parallel to the cortical surface and lower layers contain structures that are primarily perpendicular. Using this method, there was a $78.7 \pm 68 \mu \mathrm{m}$ offset in the estimate of the depth of the start of layer V. The polarity reversal method estimates the beginning of layer $\mathrm{V}$ within $\pm 90 \mu \mathrm{m}$ with $95 \%$ confidence and the intracortical stimulation method estimates it within $\pm 69.3 \mu \mathrm{m}$. We propose that these methods can be used to estimate the in situ location of laminar electrodes implanted in the rat motor cortex.
\end{abstract}

(Some figures in this article are in colour only in the electronic version)

\section{Introduction}

The development of microelectrode arrays has enabled access to disparate regions of the cortex for neurorehabilitation, neuroprosthetic and basic neuroscience research (Cogan 2008, Mercanzini et al 2008, Kipke et al 2003, Vetter et al 2004). The mammalian neocortex has a regular structure consisting of six layers that is similar across different brain areas 
(DeFelipe et al 2002). Microelectrode array technology has enabled us to span the entirety of the six layer cortex allowing simultaneous electrophysiological recordings and stimulation in different depths and layers (Kipke et al 2003, Vetter et al 2004).

One exciting application of these electrodes is in the neuroprosthetic field where neural signals are acquired from the motor cortex to control external devices (Levin et al 2000, Wessberg et al 2000, Carmena et al 2003). Previous neuroprosthetic studies have targeted neurons in the lower layers of the motor cortex (layers V and VI) (Serruya et al 2002, Taylor et al 2003, Donoghue 2002) to obtain a control signal because the large pyramidal Betz cells in these layers project to the spinal cord, and their large dipole fields result in higher recording quality relative to other cells (Humphrey et al 1970). Parikh et al have shown that units in the lower layers are significantly more likely to encode direction information as compared to units in the upper layers. Advances in electrode technology and the ability to conduct long-term, simultaneous, multi-site recordings have made it possible to evaluate event-related action potentials from different cortical layers for movement and direction information (Parikh et al 2009, Du et al 2009).

Another application of these electrodes is in intracortical microstimulation (ICMS) for neurorehabilitation applications (Taub et al 2002, Friel et al 2007). It has been shown that for specific neurorehabilitation applications it is important to target deep cortical layers (specifically layer V) (Gradinaru et al 2009). Studies on the neurorehabilitation applications of cortical electrical stimulation have shown that stimulation differentially affects neuronal activity of deep and superficial layers of the motor cortex (Yazdan-Shahmorad et al 2011). Therefore, advances in techniques to determine the location and type of cell recorded in an awake, behaving preparation via extracellular recordings will help validate proposed cortical microcircuits and the functional role of the different cells across the layered cortex (Du et al 2009).

Accurate interpretation of the signals recorded with these electrodes depends upon having a firm understanding of the neural signal and accurate placement of the electrodes (Schwartz et al 2006, Anderson et al 2007, Marzullo et al 2006, Gage et al 2005, Musallam et al 2004, Parikh et al 2009). Often, the placement of the electrodes can only be evaluated through histological and electrolytic lesioning techniques, which practically can only be done post-mortem by explanting the brain (Brozoski et al 2006, Townsend et al 2002). Although these electrodes can be visualized in situ during electrode placement at specialized institutions using MRI or CT (Larson et al 2008, Ferroli et al 2004), the small size of these electrodes and the cost of MRI and CT makes it more difficult for visualization in animal models. An in vivo method for identification of correct placement of electrodes is necessary to determine in situ electrode placement for use in the development of clinical neuroprosthetic and neurorehabilitation devices in animal models of human disorders.

In the first in vivo method for electrode localization described here, the depth of an electrode array was estimated by laminar analysis of the field potential oscillations. This method is based on the field potential polarity reversal, which appears 100-300 $\mu \mathrm{m}$ below layer IV of the cortex (Kandel and Buzsaki 1997) in laminar ketamine-xylazine-induced high voltage spindles (HVSs) of the rat cortical column. HVSs consist of a rhythmic series of spike and wave components in local field potentials (LFPs) and occur in the resting or sleeping states in rats (Kandel and Buzsaki 1997, Jando et al 1995). The intraepisodic frequency of HVSs in the anesthetized animal varies from 2 to $6 \mathrm{~Hz}$ (Kandel and Buzsaki 1997). Single fibers of thalamocortical neurons provide input mainly to layer IV but also give off collaterals to layers V and VI (Bodegreuel et al 1987, Jensen and Killackey 1987). This contributes to a polarity reversal observed $100-300 \mu \mathrm{m}$ below layer IV of the cortex (Kandel and Buzsaki 1997) which was used as a biological marker to estimate the depth of the implanted electrode in this method. The depth of polarity reversal in these oscillations was calculated and compared to the start of layer $\mathrm{V}$ based on histological analysis. The electrode recording site, in which polarity reversal appeared, was identified by estimating the instantaneous phase of each recording site using the Hilbert transform.

In the second in vivo method, the depth of the electrode array was estimated by electrically stimulating through each electrode site and comparing the minimum current that induced a threshold movement for cathodic-first versus anodicfirst pulse shapes. Previous clinical, animal and modeling studies have shown that neural elements perpendicular to the electrode surface are preferentially excited by anodic stimulation while cathodic stimulation excites those with a direction component parallel to its surface (Nitsche and Paulus 2000, Manola et al 2007, Wongsarnpigoon and Grill 2008, Yazdan-Shahmorad et al 2011). Layer V of the rat motor cortex contains large pyramidal neurons which are primarily perpendicular to the surface of the brain (Elston 2003, Brodmann 1999) and therefore parallel to the stimulating electrode sites. Upper cortical layers contain neuronal structures that are primarily parallel to the cortical surface and perpendicular to the implanted electrode surface (Brodmann 1999). Considering the structure of cortical layers and the implantation of microelectrode arrays in the rat motor cortex, we hypothesize that lower motor thresholds can be obtained by anodal stimulation in upper layers of the motor cortex compared to cathodal and vice versa in layers V/VI. To test this hypothesis, we designed an experiment to measure the motor thresholds by sequentially stimulating across different electrodes (and hence different cortical layers) of the rat motor cortex through a linear array of equally spaced electrodes.

The estimated depths from both methods were tested against the histological images. The results of both methods demonstrate placement of the electrode sites in the upper and lower cortical layers with less than $\pm 100 \mu \mathrm{m}$ error in a linear array with sites spaced $100 \mu \mathrm{m}$ apart. These results suggest that both methods are suitable for in situ electrode localization for neuroprosthetic research that has typically targeted neurons in the lower layers of the motor cortex (Serruya et al 2002, Taylor et al 2003, Donoghue 2002, Parikh et al 2009). 
(a)

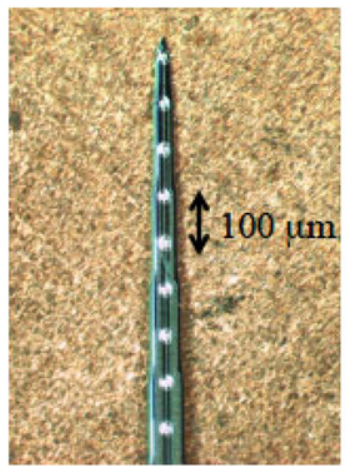

(b)

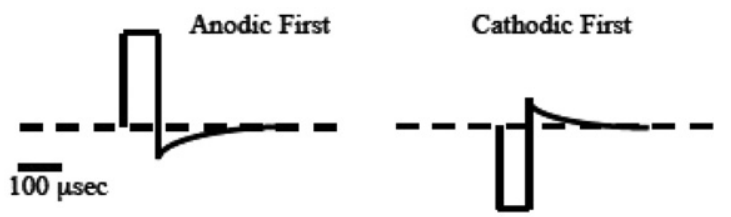

Figure 1. (a) Silicon electrode array on top of an American penny. (b) Pulse shapes: constant current CES was delivered in one of the two configurations: cathodic-first or anodic-first consisting of pulse trains. Pulses consisted of square leading phase (100 $\mu$ s) followed by an exponentially decaying second phase to balance charge. The pulse width of the leading phase was fixed at $100 \mu$ s and the length of the trailing phase was dependent upon current amplitude.

\section{Methods}

\subsection{Animal procedures}

Fourteen normal male rats weighing 275-450 g (Charles River Laboratories) were used for this study. A craniotomy was performed over the neck $(n=6)$ or forelimb $(n=$ 8) representation of the primary motor cortex (MI) in the right hemisphere. The dura was cut and folded back to allow insertion of a micro-scale penetrating electrode array (NeuroNexus Technologies) consisting of 16 electrodes linearly spaced $100 \mu \mathrm{m}$ apart (figures 1(a) and (b)) (Kipke et al 2003) with site areas of 413,703 or $1250 \mu \mathrm{m}^{2}$ on an array-by-array basis. The electrodes were inserted by hand with the aid of a dissecting microscope until the top recording site was even with the brain surface such that a maximum number of electrodes spans a six-layer neocortex. The probes were then secured using dental cement and the subjects were allowed 5-10 days to recover.

\subsection{Extracellular field potential recordings}

Extracellular field potential recordings were obtained under condition of ketamine-xylazine anesthesia. The rats were anesthetized with a mixture $\left(1.8 \mathrm{mg} \mathrm{kg}^{-1}\right)$ of ketamine $(50 \mathrm{mg}$ $\mathrm{ml}^{-1}$ ) and xylazine $\left(5 \mathrm{mg} \mathrm{ml}^{-1}\right)$. Neural electrophysiological signals were simultaneously amplified and bandpass filtered (3-90 Hz or 1-500 Hz) and sampled at 500 or $1000 \mathrm{~Hz}$ using a Multichannel Neuronal Acquisition Processor (Plexon Inc., Dallas, TX).

\subsection{Polarity reversal method}

In this method, we use the field potential polarity reversal, which appears 100-300 $\mu \mathrm{m}$ below layer IV of the cortex (Kandel and Buzsaki 1997) in laminar ketamine-xylazineinduced HVSs of the rat cortical column. The polarity reversal was found based on instantaneous phase calculations of the recorded LFPs of each electrode site. LFPs' phase estimation steps are shown in figure 2 and described as follows:

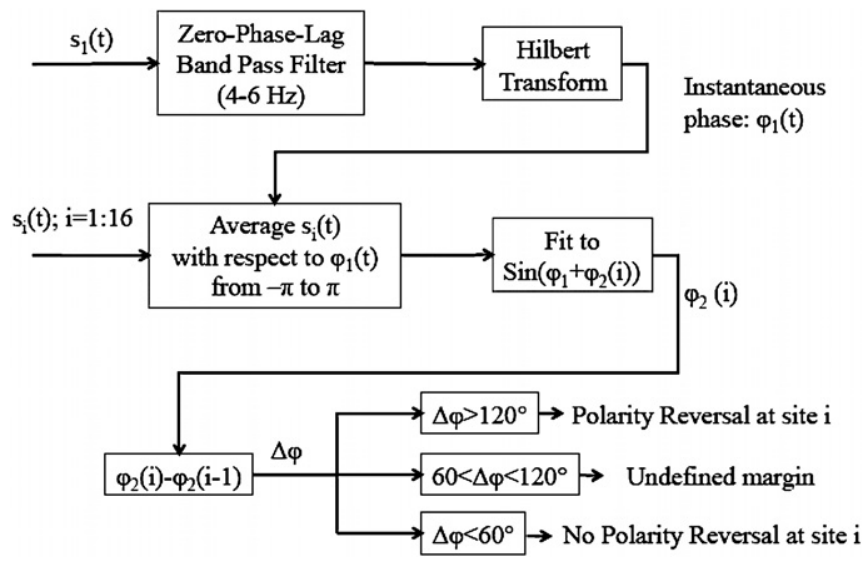

Figure 2. Block diagram of the polarity reversal method. The field potential signal of the most superficial site $\left(s_{1}(t)\right.$ : reference site) was passed through a zero-phase-lag bandpass filter $(4-6 \mathrm{~Hz})$. The Hilbert transform and therefore the instantaneous phase of the filtered signal $\left(\varphi_{1}(t)\right)$ were calculated. To calculate the phase reversal, we averaged the signal of all sites $\left(s_{i}(t) ; i=1: 16\right)$ with respect to the instantaneous phase of the most superficial signal $\left(\varphi_{1}(t)\right)$ in $-\pi$ to $+\pi$ range with $2 \pi / 100$ steps. The averages over $-\pi$ to $\pi$ phase duration were smoothed by fitting to sine waves. The phase of the smoothed signal $\left(\varphi_{2}(i)\right)$ was calculated for each recording site. The corresponding electrode sites with phase differences higher than $120^{\circ}$ are considered the polarity reversal sites.

The Hilbert transform permits a direct estimation of the instantaneous phase of a signal (Le Van Quyen et al 2001, Shaw 1996). In this method, the phase of a signal can be obtained by means of the analytic signal concept originally introduced by Gabor (1946) and recently investigated for model systems as well as for experimental data (Rosenblum et al 2001). For an arbitrary signal $s(t)$, the analytic signal $\zeta(t)$ is a complex function of time defined as

$$
\zeta(t)=s(t)+\mathrm{j} \tilde{s}(t)=A(t) \mathrm{e}^{\mathrm{j} \varphi(t)}
$$

where the function $\tilde{s}(t)$ is the Hilbert transform of $s(t)$ :

$$
\tilde{s}(t)=\frac{1}{\pi} \mathrm{P} \cdot \mathrm{V} \cdot \int_{-\infty}^{+\infty} \frac{s(t)}{t-\tau} \mathrm{d} \tau
$$

where P.V. indicates that the integral is taken in the sense of the Cauchy principal value. The instantaneous amplitude $A(t)$ and 
the instantaneous phase $\varphi(t)$ of the signal $s(t)$ are thus uniquely defined by equation (1) (Le Van Quyen et al 2001).

An important advantage of this approach is that the phase can be easily obtained for an arbitrary broadband signal. Nevertheless, application of the Hilbert transform to the unfiltered signal gives analytic phase values resembling a 'random walk'. Effective use of the Hilbert transform with LFPs must be preceded by filtering to separate the frequency band of interest from the background brain activity (Freeman 2004, 2005, 2006).

To capture the polarity reversal appearing in the ketaminexylazine-induced field potential oscillations, corresponding to each electrode site, the field potential signal of the most superficial site $\left(s_{1}(t)\right.$ : reference site) was passed through a zero-phase-lag bandpass filter (4-6 Hz) (Kandel and Buzsaki 1997). The Hilbert transform, and therefore the instantaneous phase of the filtered signal $\left(\varphi_{1}(t)\right)$ were calculated (figure 2). To calculate the phase reversal, we averaged the LFPs of all sites $\left(s_{i}(t) ; i=1: 16\right)$ with respect to the instantaneous phase of the most superficial signal $\left(\varphi_{1}(t)\right)$ in $-\pi$ to $+\pi$ range with $2 \pi / 100$ steps (figure 2 ). The averages over $-\pi$ to $\pi$ phase duration were smoothed by fitting to sine waves. The phase of the smoothed signal $\left(\varphi_{2}(i)\right)$ was calculated for each recording site. The highest phase difference corresponds to the site of polarity reverse. Since we are expecting to see a polarity reversal at this site, the phase difference should be about $180^{\circ}$. We have considered a $60^{\circ}$ margin for the polarity reversal. Any phase difference less than $60^{\circ}$ is considered not indicative of a polarity reversal.

To demonstrate the independence of our method to the referenced (most superficial) site, a virtual depth experiment was designed in which the most superficial site was changed from site 1 progressively to site 16 and the polarity reversal site was recalculated based on the method described above.

\subsection{Intracortical stimulation method}

For the second proposed method, the depth of the electrode array is estimated by stimulating through each electrode site and comparing the minimum current that induces a movement for cathodic-first versus anodic-first pulse shapes. Constant current intracortical simulation was delivered in one of the two configurations: cathodic-first or anodic-first consisting of $1 \mathrm{~s}$ pulse trains at the frequency of $100 \mathrm{~Hz}$. Pulses consisted of square leading phase $(100 \mu \mathrm{s})$ followed by an exponentially decaying second phase to balance charge of length dependent upon the amplitude of the leading phase. Current-induced movements (CIMs) were determined as the weakest current passed through the cortical electrode that caused a forced movement in 50\% of test pulses (Teskey et al 2003, AdkinsMuir and Jones 2003, Brown et al 2006, Plautz et al 2003). The anodic and cathodic CIMs then were compared. In cases in which anodic CIM was higher than cathodic CIM it was concluded that the corresponding electrode site was located in upper cortical layers (I-IV). On the other hand, when anodic CIM was lower than cathodic CIM, it was concluded that the electrode site was located in the lower cortical layers (V-VI). In cases in which anodic CIM was equal to cathodic CIM, it was concluded that no information on cortical location could be obtained.

\subsection{Comparing two methods}

To compare these methods, the depth of layer $\mathrm{V}$ for each animal was estimated based on the data from all other animals. In this comparison we evaluated the reliability of each method by assuming that we have just implanted that animal and estimated the depth of layer $\mathrm{V}$ based on each method proposed without a priori knowledge of that animal's histology. This was done by leave-one-out cross-validation (LOOCV). Cross-validation involves partitioning a sample of data into complementary subsets, performing the analysis on one subset (called the training set), and validating the analysis on the other subset (called the validation set or testing set). LOOCV uses a single animal from the original sample as the validation data, and the remaining animals as the training data (Picard and Cook 1984). This is repeated such that each observation in the sample is used once as the validation data.

\subsection{Histology}

Upon completion of the experiment, electrolytic lesions were made followed by histological analysis to determine the electrode site locations within the different cortical layers (Parikh et al 2009). Three electrode sites that were approximately at the top, middle and bottom of each electrode array that had low impedances were chosen for lesioning. At these selected sites we passed $35 \mu \mathrm{A}$ dc for $2 \mathrm{~s}$ using a potentiostat (AUTOLAB, EcoChemie, The Netherlands) to create micro-lesions (Parikh et al 2009). Animals were deeply anesthetized before lesioning. Lesion marks, on average, were 40,60 and $70 \mu \mathrm{m}$ in diameter for electrode site diameters of 23 , 30 and $40 \mu \mathrm{m}$. Serial $100 \mu \mathrm{m}$ coronal slices were stained with a standard cresyl-violet (Nissl) staining method (figures 3(c)(e)). The slices were then analyzed under a microscope and images were taken to reconstruct the position of the electrode array based on the shank track, centroid of the lesion marks, and the known geometry of the probe. In all cases, electrodes extracted from the brain were intact and were kept attached to the skull/headcap. The angle, location and length of the intact electrodes were calculated through the images taken from these headcaps (figures 3(a) and (b)). To make a more precise estimation of the probe location and angle, exact stereotaxic positions of lesion marks and probe tracts were identified by co-registering the results of histological image analysis to the estimated probe locations from the images taken from the intact arrays (Parikh et al 2009). After determining the location of the electrode array, we identified the location of the boundary between the upper and lower layers (the start of layer V) by analyzing the gray-level index values of the images using ImageJ (RSB, NIH: http://rsb.info.nih.gov/ij/). At the beginning of layer $\mathrm{V}$, the gray-level index increases abruptly due to the large pyramidal cells and the higher cell body density (Parikh et al 2009).

\section{Results}

\subsection{Polarity reversal method}

The results of each analysis step for one animal (M2) are shown in figure 4. Figure 4(a) shows the ketamine-xylazine-induced 

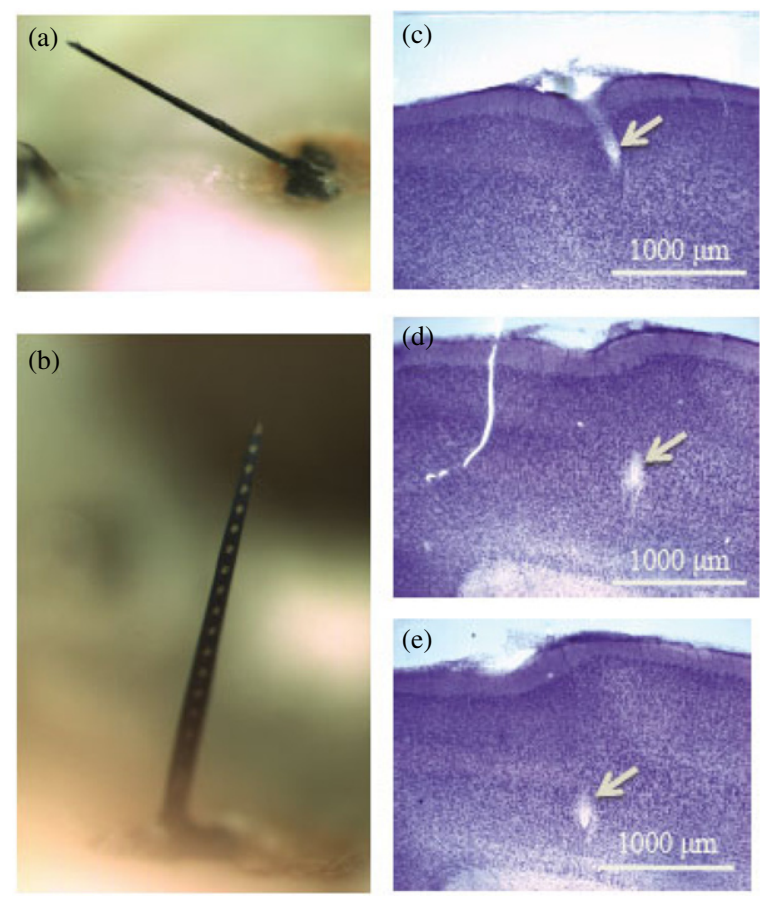

Figure 3. (a) and (b) The angle and depth of the silicon probe was estimated using these images of intact skulls/headcaps. Individual electrodes can be seen along the silicon shank in (b). (c)-(e)

Histology images from one rat. Three lesion marks are shown at the different depths where the electrode was implanted. The lesion marks are used to reconstruct the trajectory of the silicon probe and depth of layer $\mathrm{V}$ relative to each site in the array.

field potential oscillations recorded from the motor cortex. Sites are arranged such that site 1 is located closest to the cortical surface. The peak amplitude shown on top sites (1, $2,3)$ starts to decrease and it disappears completely at site 6. Figure 4(b) shows the average of filtered LFPs with respect to the first site of the recording electrode (site 1). In figure 4(c) the phase of each of the recording sites for a sample recording $(\varphi 2(i))$ is shown. These data demonstrate a clear phase shift that occurs between sites 4 and 5 for this rat. Figure 4(d) shows the phase differentiation from site to site (differentiation of plot 4c). The highest amplitude corresponds to site 5 for this rat. This site is used to determine the depth of layer $\mathrm{V}$ in this method.

The results of our analysis for the polarity reversal method for all animals are listed in table 1 . We found polarity reversal in LFPs recorded in 7 of 8 rats. For animal M4, we did not find a polarity reversal in the recorded LFPs as the most superficial electrode was located deeper than the start of layer $\mathrm{V}$. The average depth of polarity reversal was calculated to be $928.4 \pm 110.4 \mu \mathrm{m}$. The offset is calculated based on the start of layer $\mathrm{V}$ from histological analysis (table 1). Our results show that polarity reversal appears $150.1 \pm 104.2 \mu \mathrm{m}$ below the start of layer V.

The results of changing the reference site in a virtual depth experiment from site 1 (the most superficial) to site 16 (the deepest) are shown in figure 5. Changing the reference (most superficial) site from site 1 to the site demonstrating the polarity reversal showed the previously detected polarity reversal site as the site in which we see a phase difference higher than $120^{\circ}$. We therefore determined this site to be the most accurate polarity reversal site. Changing the reference site from the polarity reversal site to site 16 , being the deepest electrode in the array relative to the surface of the cortex, showed no polarity reversal. These results demonstrate the independence of our phase calculating method from the reference site.

\subsection{Intracortical stimulation method}

The results of the intracortical stimulation method are summarized in figure 6 and table 2. Figure 6 shows the anodic-first and cathodic-first CIM threshold difference as a
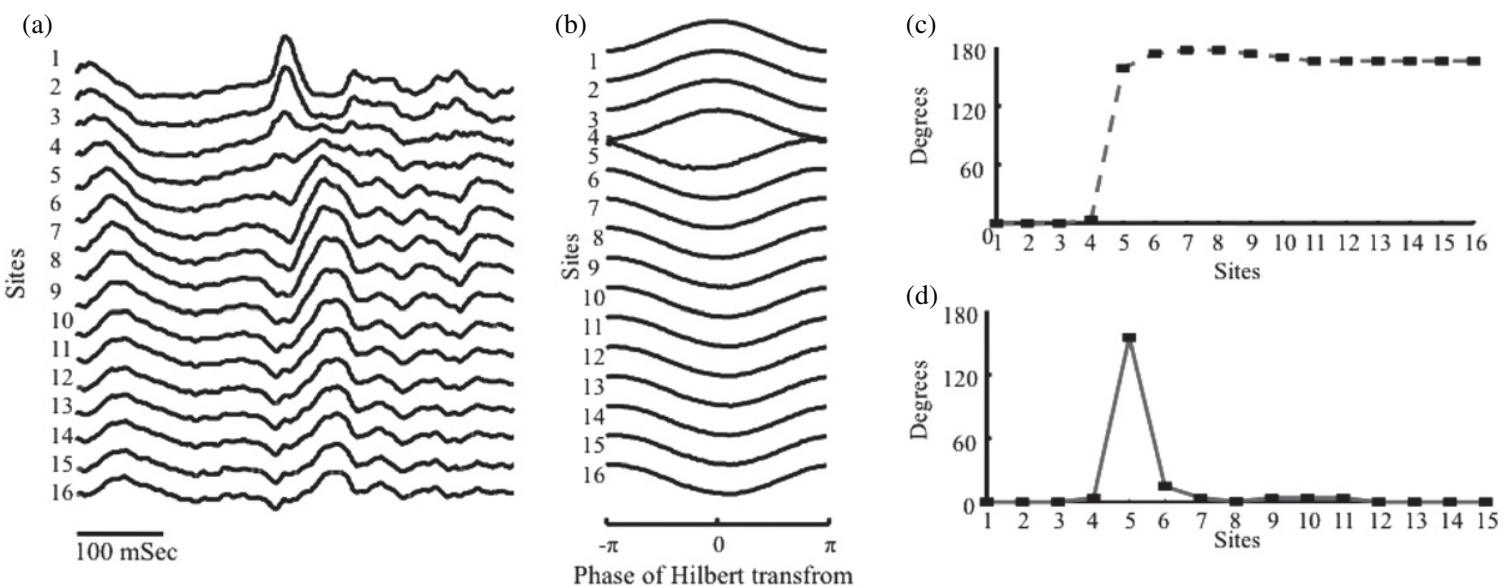

Figure 4. (a) 16 channel ketamine-xylazine-induced field potential oscillations recorded from motor cortex of a single rat. The peak amplitude shown on top sites $(1,2,3)$ starts to decrease and it disappears completely at site 6 . Sites are arranged such that site 1 is located closest to the cortical surface. (b) Average of filtered LFPs with respect to the first site of the recording electrode (site 1). (c) Phase of each of the recording sites for a sample recording. These data demonstrate a clear phase shift that occurs between sites 4 and 5 . (d) Phase differentiation from site to site (differentiation of plot c). The highest amplitude corresponds to site 5 for this rat (M2 in table 1). This site is used to determine the depth of layer V in the polarity reversal method. Reproduced from Yazdan-Shahmorad et al (2007) with permission (C) 2007 IEEE). 


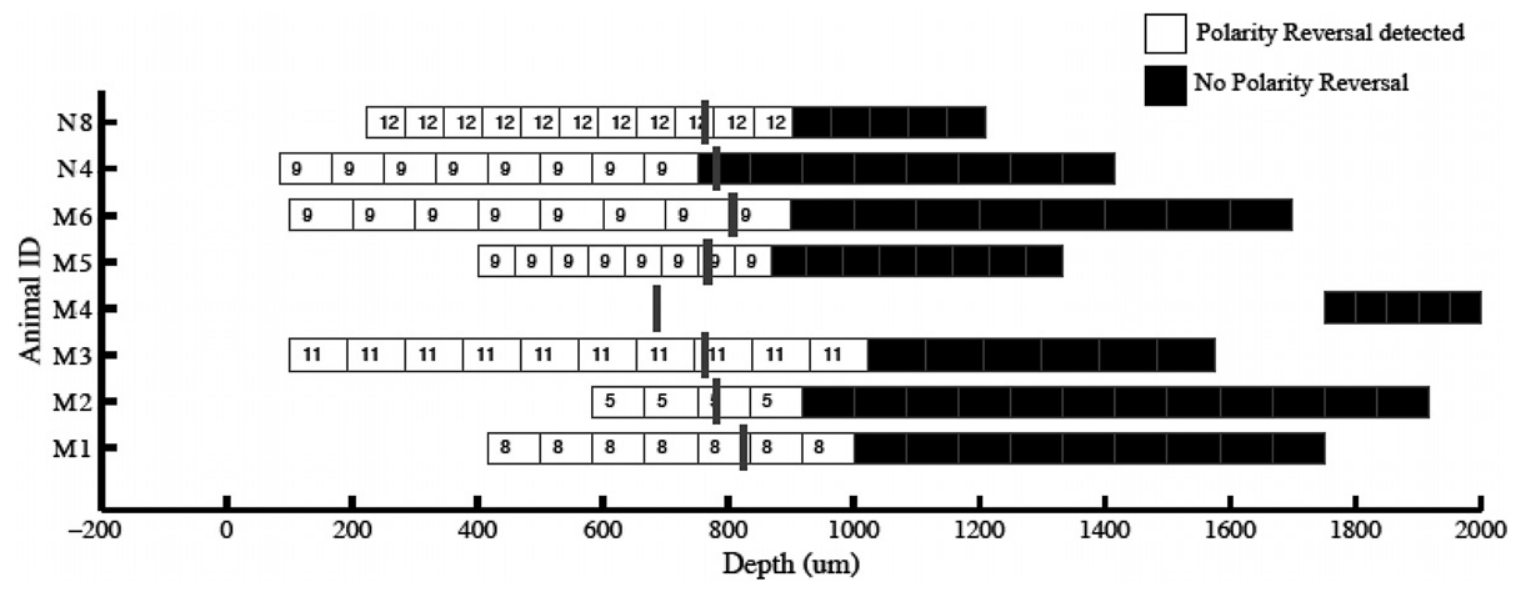

Figure 5. Virtual depth experiment: for each electrode, the reference site was changed from 1 to 16 (site 1 being closest to the cortical surface) and the polarity reversal was calculated. Each square depicts one electrode site in the array. The widths of squares are consistent within each 'array' and are based on the angle of the electrode relative to the layered cortex. White squares show that polarity reversal was detected with respect to the sites located deeper. The number on that square indicates the site number of the polarity reversal. Black squares indicate that no polarity reversal was detected by choosing those sites as reference with respect to the sites located deeper. The gray bar indicates the depth of layer V as determined through histology. In animal M4, no polarity reversal was observed as every electrode in this array was located deeper than layer V.

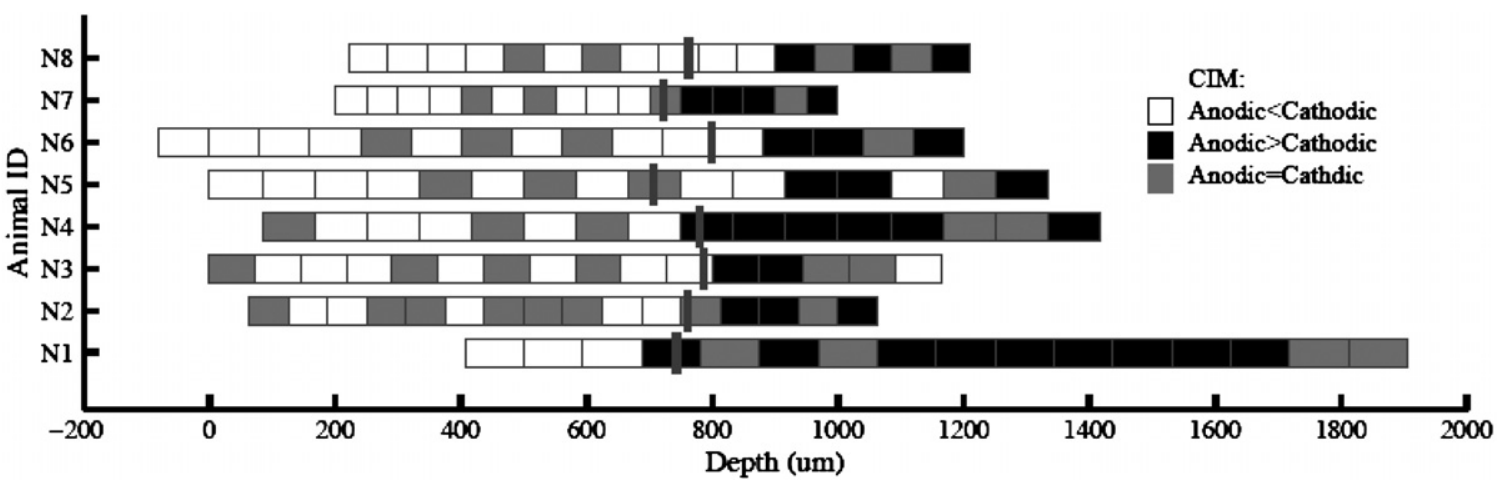

Figure 6. Anodic and cathodic CIM threshold differences: each square indicates the threshold for inducing a movement with anodic-first intracortical stimulation relative to cathodic-first stimulation. The widths of the squares are consistent within each 'array' and are based on the angle of the electrode relative to the layered cortex. As one moves from superficial electrodes (left) to deep electrodes (right), the threshold for inducing a movement with anodic-first stimulation becomes higher than the threshold for inducing movement with cathodic-first stimulation. The location of this change is used to estimate the location of layer $\mathrm{V}$ with the intracortical stimulation method. The gray bar indicates the depth of layer $\mathrm{V}$ as determined through histology.

Table 1. Results based on histology and polarity reversal method. Averages indicate mean \pm SD. Column C shows the offset of the estimation of depth of layer $\mathrm{V}$ for this method in comparison with histological analysis. The polarity reversal was exclusively observed below layer V. The estimate of the polarity reversal (E) was obtained with the LOOCV method for each validation animal, calculated from the training data. The error of the LOOCV method $(\mathrm{F})$ is the difference between the estimate and the actual location of layer $\mathrm{V}$ based on histology. Data are in $\mu \mathrm{m}$.

\begin{tabular}{|c|c|c|c|c|c|c|c|}
\hline $\begin{array}{l}\text { Animal } \\
\text { ID }\end{array}$ & $\begin{array}{l}\text { Polarity } \\
\text { reversal (site) }\end{array}$ & $\begin{array}{l}\text { (A) Depth of } \\
\text { polarity reversal }\end{array}$ & $\begin{array}{l}\text { (B) Depth of layer } \\
\text { layer V (histology) }\end{array}$ & $\begin{array}{l}\text { (C) Offset } \\
(\mathrm{A}-\mathrm{B})\end{array}$ & $\begin{array}{l}\text { (D) Mean of offset for } \\
\text { the training dataset }\end{array}$ & $\begin{array}{l}\text { (E) Estimate of } \\
\text { layer V }(A-D)\end{array}$ & $\begin{array}{l}\text { (F) Error } \\
(\mathrm{E}-\mathrm{B})\end{array}$ \\
\hline M1 & 8 & 1101 & 819 & 282 & 128 & 973 & 153 \\
\hline M2 & 5 & 842 & 775 & 67 & 164 & 678 & -97 \\
\hline M3 & 11 & 1052 & 758 & 294 & 126 & 926 & 167 \\
\hline M4 & No PR & - & 680 & - & 150 & - & - \\
\hline M5 & 9 & 903 & 762 & 141 & 151 & 752 & -10 \\
\hline M6 & 9 & 910 & 802 & 108 & 157 & 753 & -49 \\
\hline N4 & 9 & 791 & 776 & 15 & 172 & 619 & -157 \\
\hline N8 & 12 & 901 & 757 & 144 & 151 & 750 & -7 \\
\hline Average & & $928.6 \pm 110.4$ & $766.1 \pm 41.1$ & $150.1 \pm 104.2$ & $\begin{array}{r}150.1 \pm 16.1 \\
95 \% \mathrm{conf}\end{array}$ & $\begin{array}{l}778.4 \pm 127.4 \\
\text { ence interval: } \pm 9\end{array}$ & SD: \pm 121.5 \\
\hline
\end{tabular}



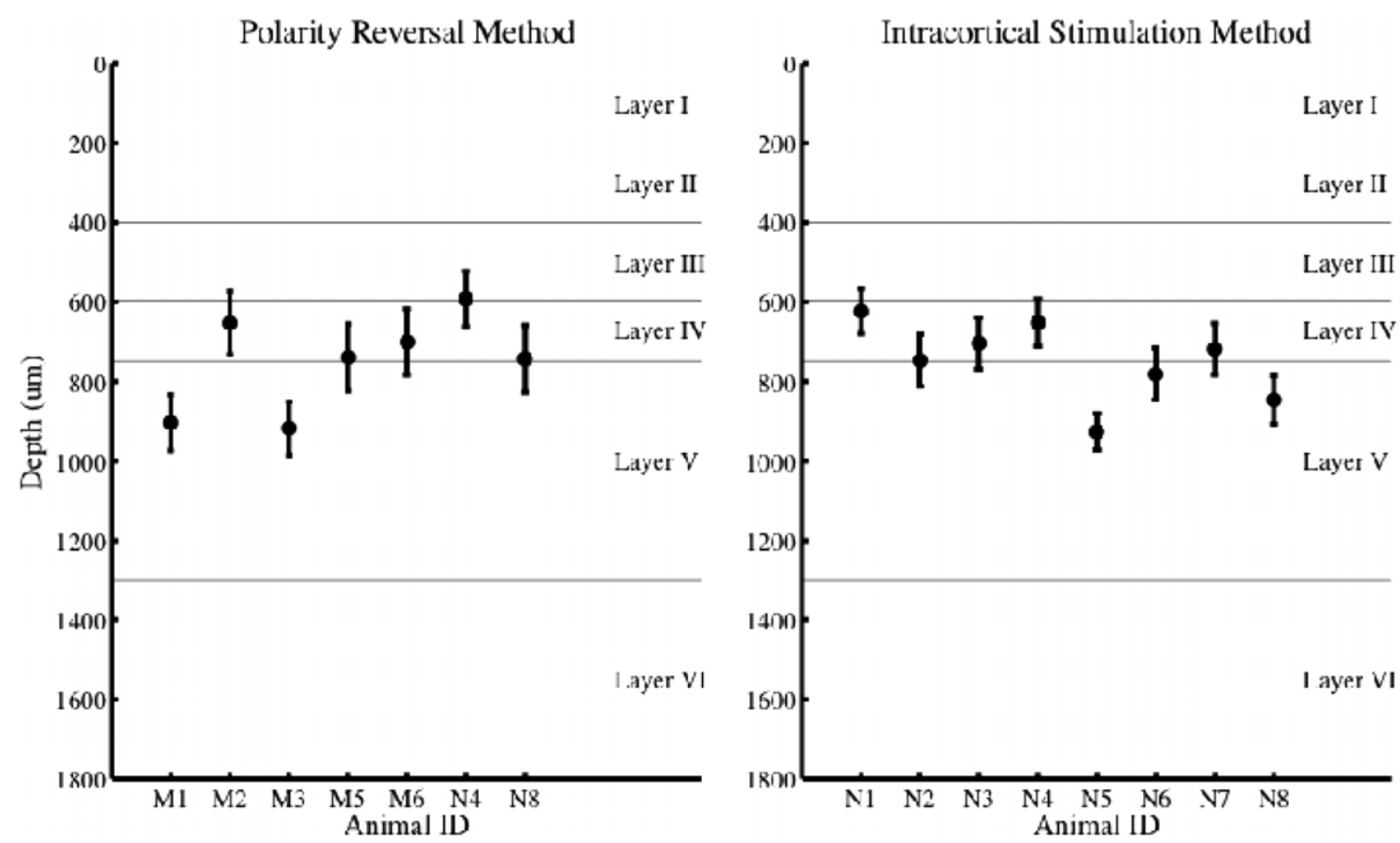

Figure 7. In situ estimation of layer $\mathrm{V}$ based on the polarity reversal method (left) and intracortical stimulation method (right). Data are plotted on a schematic of the layered rat motor cortex with relative distances between layers (Skoglund et al 1997). The markers are the estimation of layer $\mathrm{V}$ as reported in column $\mathrm{F}$ of tables 1 and 2 without a priori knowledge of the depth of layer $\mathrm{V}$. The bars show the $95 \%$ confidence interval based on the dataset excluding the representative animal from the dataset using the LOOCV method.

Table 2. Results based on histology and the intracortical stimulation method. Averages indicate mean \pm SD. Column C shows the offset of the estimation of depth of layer $\mathrm{V}$ for this method in comparison with histological analysis. A negative offset indicates the movement threshold change occurred superficial to layer V. The estimate of the CIM change (E) was obtained with the LOOCV method for each validation animal, calculated from the training data. The error of the LOOCV method $(\mathrm{F})$ is the difference between the estimate and the actual location of layer $\mathrm{V}$ based on histology. Data are in $\mu \mathrm{m}$.

\begin{tabular}{|c|c|c|c|c|c|c|c|}
\hline $\begin{array}{l}\text { Animal } \\
\text { ID }\end{array}$ & $\begin{array}{l}\text { CIM change } \\
\text { (site) }\end{array}$ & $\begin{array}{l}\text { (A) Depth of CIM } \\
\text { change }(\mu \mathrm{m})\end{array}$ & $\begin{array}{l}\text { (B) Depth of layer } \\
\text { V (histology) }\end{array}$ & $\begin{array}{l}\text { (C) offset } \\
(\mathrm{A}-\mathrm{B})\end{array}$ & $\begin{array}{l}\text { (D) Mean of offset } \\
\text { for the training dataset }\end{array}$ & $\begin{array}{l}\text { (E) Estimate of } \\
\text { layer V }(A-D)\end{array}$ & $\begin{array}{l}\text { (F) Error } \\
(\mathrm{E}-\mathrm{B})\end{array}$ \\
\hline N1 & 4 & 687 & 738 & -51 & 75 & 612 & -126 \\
\hline N2 & 13 & 812 & 755 & 57 & 59 & 753 & -3 \\
\hline N3 & 12 & 800 & 781 & 19 & 65 & 735 & -47 \\
\hline N4 & 9 & 750 & 776 & -26 & 71 & 679 & -98 \\
\hline N5 & 12 & 916 & 702 & 214 & 37 & 879 & 176 \\
\hline N6 & 13 & 880 & 793 & 87 & 55 & 825 & 31 \\
\hline N7 & 12 & 750 & 718 & 32 & 63 & 687 & -32 \\
\hline N8 & 12 & 901 & 757 & 144 & 47 & 854 & 96 \\
\hline Average & & $812 \pm 81.8$ & $752.5 \pm 31.6$ & $59.5 \pm 87.6$ & $\begin{array}{l}59.5 \pm 12.5 \\
95 \% \text { confidence interva }\end{array}$ & $\begin{array}{l}752.5 \pm 93.6 \\
: \pm 69.3\end{array}$ & SD: \pm 99.9 \\
\hline
\end{tabular}

function of cortical depth. Table 2 lists the electrode site number and depth of the CIM threshold change for anodic and cathodic stimulation. The threshold change indicates the electrode in the array at which anodic-first intracortical stimulation had a higher threshold for eliciting a movement than cathodic-first stimulation. The average estimated depth of CIM threshold change based on this method was $812 \pm$ $81.8 \mu \mathrm{m}$, corresponding to the $687-916 \mu \mathrm{m}$ start of layer $\mathrm{V}$. The offset is calculated based on the difference of the depth of CIM threshold change and the histological indication of the depth of layer V. Our results show that CIM change appears with a $78.8 \pm 68 \mu \mathrm{m}$ offset below the start of layer V.

\subsection{Comparing two methods}

We evaluated the reliability of each method by LOOCV described above. We used the data from a single animal as the validation data, and the remaining animals as the training data. This was repeated such that the data from each animal from the dataset were used once as the validation data (each row in tables 1 and 2). We used the average of the offset (column C) from tables 1 and 2 from all of the animals excluding the validation animal as the training data. This average is shown in column (D) in these tables. This column is the average offset for each method in comparison to the start of layer $\mathrm{V}$ for each training set. To estimate the depth of layer $\mathrm{V}$ for each method we subtracted this offset from the depth of the 
biological marker (column A) used for each method (column E). To calculate the error of each LOOCV we compared these estimations with the histology results (column F). The estimation results, along with the $95 \%$ confidence intervals in the estimation, are shown in figure 7 and represent the estimate of layer $\mathrm{V}$ without a priori knowledge of the actual depth of layer V. These results indicated that we can estimate the depth of the start of layer $\mathrm{V}$ within $\pm 90 \mu \mathrm{m}$ for the polarity reversal method and $\pm 69 \mu \mathrm{m}$ for the intracortical stimulation method with $95 \%$ confidence relative to the physical location of the start of layer $\mathrm{V}$.

In two animals $(\mathrm{N} 4, \mathrm{~N} 8)$, both methods were used for estimating the depth of the start of layer V. The depth of polarity reversal estimated for N4 was $619 \mu \mathrm{m}$ which was $157 \mu \mathrm{m}$ above the beginning of layer $\mathrm{V}(757 \mu \mathrm{m})$, while the depth of CIM threshold change for this rat was estimated to be $679 \mu \mathrm{m}, 98 \mu \mathrm{m}$ above the start of layer V. Alternatively, the depth of polarity reversal estimated for $\mathrm{N} 8$ was $750 \mu \mathrm{m}$ which was located $7 \mu \mathrm{m}$ above the start of layer $\mathrm{V}(757 \mu \mathrm{m})$, while the depth of MT change for this rat was estimated to be $854 \mu \mathrm{m}, 96 \mu \mathrm{m}$ below the start of layer V. Both of these methods, when used together in these animals, predicted the depth of the beginning of layer $\mathrm{V}$ within the $95 \%$ confidence intervals of both methods.

\section{Discussion}

\subsection{Electrophysiological-based laminar analysis}

The polarity reversal of cortical field potentials appears 100$300 \mu \mathrm{m}$ below layer IV of the motor cortex (Kandel and Buzsaki 1997). The motor cortex in the rat has a small but distinct layer IV $(\sim 100 \mu \mathrm{m}$ thick $)$ which receives thalamic inputs and begins around $650 \mu \mathrm{m}$ and ends around $750 \mu \mathrm{m}$ below the surface of the brain (Skoglund et al 1997). Therefore we expect to find the polarity reversal at a depth of 850$1050 \mu \mathrm{m}$. Our results showed that the average depth was calculated to be $928.4 \pm 110.4 \mu \mathrm{m}$, which lies in the reported range of polarity reversal in the cortical column for HVS (Kandel and Buzsaki 1997). Also, as reported in table 1, this method can estimate the depth of the start of layer $\mathrm{V}$ within $\pm 90 \mu \mathrm{m}$ with $95 \%$ confidence. As electrode recording sites in the probes used in this project were spaced at $100 \mu \mathrm{m}$, this gives us a laminar resolution of $100 \mu \mathrm{m}$ along the $1500 \mu \mathrm{m}$ shank and allows us to tolerate up to $50 \mu \mathrm{m}$ error in polarity reversal and structural depth estimation with respect to the expected range for each method. Recording electrodes with lower site spacing may give more laminar resolution, reduce the error and provide a more accurate estimation.

No polarity reversal was found for animal M4 by calculating the phase difference of its electrode recording sites. Considering the electrode configuration, we concluded that the electrode was placed so deep in the motor cortex that the first site was past the polarity reverse point (beginning of layer $\mathrm{V}$ ). Histological analysis showed that the depth of the first site for this rat was $1826 \mu \mathrm{m}$ below the cortical surface, which confirms our prediction.

As described in the text, the independence of our method from the referenced site was evaluated. The results showed that the phase difference of the HT of each electrode site and the referenced site is high $\left(>120^{\circ}\right)$ when the referenced site is located superficial to the polarity reversal and low $\left(<60^{\circ}\right)$ when the referenced site is located deeper than the polarity reversal. This demonstrates the robustness of the polarity reversal method due to the independence of the referenced site.

\subsection{Intracortical stimulation}

In the intracortical stimulation method, the depth of the electrodes in the array is estimated by comparing the minimum current that induces a movement for cathodic-first versus anodic-first pulse polarity. This method is based on previous studies that show that neural elements perpendicular to the electrode surface are preferentially excited by anodic stimulation while cathodic stimulation excites those with a direction component parallel to its surface (Nitsche and Paulus 2000, Manola et al 2007, Wongsarnpigoon and Grill 2008). Upper cortical layers contain the neuronal structures that are primarily parallel to the cortical surface while lower layers of the rat motor cortex contain large pyramidal neurons which are primarily perpendicular to the surface of the brain (Brodmann 1999). We used this stimulation method to detect the structural change that appears at the beginning of layer V.

Previous investigations on the effects of extracellular anodic and cathodic stimulation on cortical neurons have inferred that the differences obtained are due to the opposing membrane potential changes induced between oppositely directed poles (dendrite and axon) of the neurons (Bishop and Oleary 1950, Creutzfeldt et al 1962, Hern et al 1962, Landau et al 1964, Libet and Gerard 1941). Upper cortical layers contain the neuronal structures that are primarily parallel to the cortical surface and therefore perpendicular to the stimulating electrode surface (Brodmann 1999). When stimulating through the perpendicularly implanted microelectrode array in these layers, anodic current is thought to hyperpolarize the dendrites while depolarizing cell body and axonal portions of neurons located in these layers. An opposite sequence of depolarizing-hyperpolarizing events is thought to occur during cathodic current flow (Gorman 1966). Therefore it is expected to see lower anodic currents to induce movements in these layers in comparison with cathodic currents.

Previous modeling studies have suggested that cathodic stimulation excites the neural elements with a direction component parallel to the surface of stimulation (Manola et al 2007, Wongsarnpigoon and Grill 2008). The layer V of the rat motor cortex contains large pyramidal neurons which are primarily perpendicular to the surface of the brain (Elston 2003, Brodmann 1999) and therefore parallel to the stimulating electrode sites of the implanted microelectrode array. We expected to see lower cathodic currents to induce movements in layer $\mathrm{V}$ in comparison with anodic currents. In addition, it has been reported that the site of excitation is dependent on the polarity of the stimulus, with cathodic stimuli resulting in lower thresholds for electrode positions closer to the axon and anodic stimuli resulting in lower thresholds for electrode positions closer to the cell body and dendrites (Yeomans et al 
1988, Matthews 1977, McIntyre and Grill 1999). Pyramidal cell neurons located within layer $\mathrm{V}$ in the primary motor cortex send their axons down to the spinal cord. They have apical, perisomatic and basal dendrites that project into all cortical layers (Elston 2003, Franceschetti et al 1998). Therefore, their dendrites are located in upper layers (I-IV), while their axons are located in lower layers (V-VI) of motor cortex. This will also contribute to our hypothesis of having lower CIM for cathodic stimulation in these layers in comparison with anodic stimulation. Our results from the intracortical stimulation method agree with these previous findings and support our hypothesis.

Since, on average, layer $\mathrm{V}$ of the cortex starts $750-800 \mu \mathrm{m}$ below the surface of the cortex (Skoglund et al 1997), we therefore expect to see a change in the difference of anodic and cathodic CIMs in this range due to the large neural elements of pyramidal cells traveling the depth of a cortical column. The depth of CIM change in our results was in the range of $812 \pm 81.8 \mu \mathrm{m}$ below the surface of the brain, which is below the expected range. As reported in table 2, this method can estimate the depth of the start of layer V to within $\pm 69.3 \mu \mathrm{m}$ with $95 \%$ confidence. In this method, recording electrodes with lower site spacing may give us more laminar resolution, reduce the error and provide a more accurate estimation.

The high error in some cases in our results can be explained in part by the angle of the electrode in the brain relative to the organization of the layered cortex. We attempted to implant the electrodes as perpendicular to the surface of the brain as possible; however, in some cases they were implanted at an angle. Because this method is sensitive to the orientation of the electrode site surface with respect to the orientation of fibers, this angle can cause additional errors in the depth estimation. As has been suggested by Wongsarnpigoon and Grill, to predict the response of the neural elements located at different angles with respect to the stimulation surface, further modeling studies need to be conducted. However, because our results are also described by the site of excitation in addition to the direction of the neuronal fibers, this error will be limited when the stimulation electrode site is proximate to the axonal parts of layer V neurons (Nowak and Bullier 1998a, 1998b, Yeomans et al 1988, Matthews 1977, McIntyre and Grill 1999).

\subsection{Comparing the two methods}

The polarity reversal method gives a $90 \mu \mathrm{m}$ confidence interval for the estimation of the start of layer $\mathrm{V}$, while the intracortical stimulation method gives a $69 \mu \mathrm{m}$ confidence interval, suggesting that the intracortical stimulation method can provide a more accurate estimate.

In two animals (N4 and N8), we were able to apply both methods for localizing layer $\mathrm{V}$. The polarity reversal method gave a more accurate estimation for N8 while the intracortical stimulation method gave a more accurate estimation for N4. This could be explained in part by the large angle of the electrode array N8 in comparison to N4 relative to the cortical layers. As the hypothesis behind the intracortical stimulation method is based on the direction of the cortical fibers, this method is more sensitive to the angle of the electrode compared to the polarity reversal method. We can conclude that in cases in which we have a priori knowledge of the electrode array implanted at an angle, the polarity reversal method will give a more accurate estimation of the depth of the start of layer V.

Although we consider the neocortex in many species and cortical areas to be built according to a stereotypic cortical architecture (Braitenberg and Schuz 1991), it has to be taken into consideration that there are certain variations between different cortical areas in both architecture and particularly in the layout of horizontal connections (Lund et al 1993). Furthermore, across the cortical surface, within and/or between neighboring areas, distinct discontinuities of horizontal connections exist (Manger et al 1997). Such variability might affect the biological markers in our proposed methods and deserves attention in each case in which these methods are used. Nevertheless, in view of a common architecture of neocortex, the present results obtained in the rat motor cortex provide reliable methods for estimating the depth of the implanted linear electrode array for other cortical areas and/or species.

\subsection{Accuracy of the histological localization and layer estimation}

The depth of the beginning of layer $\mathrm{V}$ was calculated with the program ImageJ which has a high accuracy for identifying the change in light density of nissl-stained images associated with the start of dense pyramidal cell bodies in layer V (Parikh et al 2009). Given the micro-scale of these electrode arrays, it is necessary to make three lesion marks along the shank to get accurate electrode placement by aligning a three-dimensional vector between 'points' indicating the lesion. Therefore, it is likely that the majority of the errors recorded in these measurements are attributed to the limitations of each of the individual methods rather than localizing the electrodes relative to layer V. While the centroid of each electrode was spaced $100 \mu \mathrm{m}$ apart, the addition of the diameter of each electrode meant that the true distance between electrodes was 77,70 and $60 \mu \mathrm{m}$ for electrode site diameters of 23 , 30 and $40 \mu \mathrm{m}$, respectively. Ideally, each electrode would be an infinitely small 'point-source' relative to the size of the neural elements being measured or activated in order to make a precise measurement. However, each of the methods described here involves the activity of more than just one neuron or neural element. In the phase reversal method, we are recording changes in the local electrical fields. These fields are highly susceptible to the orientation and type of neural element coursing through each of the neural layers that are a likely source of error in this method. In the CIM method, charge is activating a number of output neurons innervating muscle to affect an easily observable muscle 'twitch'. The size and shape of the electric fields generated at the stimulated electrode are likely complex and have a degree of overlap between neighboring electrodes. It remains to be seen if reducing the diameter and spacing of the electrodes will improve the accuracy of these techniques or if the error inherent to each 
technique is too great for further improvement. Ultimately, these techniques achieve the goal of identifying the electrode in an array closest to the output layer of motor cortex and thus most effective for neuroprosthetics and neurorehabilitation research.

\section{Conclusion}

In this study we propose and compare two in vivo methods for the estimation of electrode depth in the rat primary motor cortex in situ. The results of both methods demonstrate their utility to define the placement of the electrode sites in the upper and lower cortical layers. The proposed methods are reliable candidates for targeting deep and superficial layers within three electrodes in an array of electrodes spaced $100 \mu \mathrm{m}$ spanning the layered motor cortex. This has important implications for neuroprosthetic and neurorehabilitation research that has typically targeted neurons in the lower layers of the motor cortex (Serruya et al 2002, Taylor et al 2003, Donoghue 2002, Parikh et al 2009, Du et al 2009).

\section{Acknowledgment}

The authors acknowledge E Kim for her contribution in histology preparations and are grateful for informative discussions with J Berke and E Ionides.

\section{References}

Adkins-Muir D L and Jones T A 2003 Cortical electrical stimulation combined with rehabilitative training: enhanced functional recovery and dendritic plasticity following focal cortical ischemia in rats Neurol. Res. 25 780-8

Anderson W, Kudela P, Cho J, Bergey G and Franaszczuk P 2007 Studies of stimulus parameters for seizure disruption using neural network simulations Biol. Cybern. 97 173-94

Bishop G H and Oleary J L 1950 The effects of polarizing currents on cell potentials and their significance in the interpretation of central nervous system activity Electroencephalogr. Neurophysiol. 2 401-16

Bodegreuel K M, Singer W and Aldenhoff J B 1987 A current source density analysis of field potentials-evoked in slices of visual-cortex Exp. Brain Res. $69213-19$

Braitenberg V and Schuz A 1991 Anatomy of the Cortex: Statistics and Geometry (New York: Springer)

Brodmann K 1999 Brodmann's Localisation in the Cerebral Cortex/Translated with Editorial Notes and an Introduction ed L J Garey (London: Imperial College)

Brown J A, Lutsep H L, Weinand M and Cramer S C 2006 Motor cortex stimulation for the enhancement of recovery from stroke: a prospective, multicenter safety study Neurosurgery $\mathbf{5 8} 464$

Brozoski T J, Caspary D M and Bauer C A 2006 Marking multi-channel silicon-substrate electrode recording sites using radiofrequency lesions J. Neurosci. Methods 150185

Carmena J M, Lebedev M A, Crist R E, O’Doherty J E, Santucci D M, Dimitrov D F, Patil P G, Henriquez C S and Nicolelis M A 2003 Learning to control a brain-machine interface for reaching and grasping by primates PLoS Biol. 1 193-208

Cogan S F 2008 Neural stimulation and recording electrodes Annu. Rev. Biomed. Eng. 10275

Creutzfeldt O, Fromm G H and Kapp H 1962 Influence of transcortical D-C current on cortical neuronal activity Exp. Neurol. 5 436-52
DeFelipe J, Alonso-Nanclares L and Arellano J I 2002 Microstructure of the neocortex: comparative aspects J. Neurocytol. 31 299-5

Donoghue J P 2002 Connecting cortex to machines: recent advances in brain interfaces Nature Neurosci. 5 1085-8

Du J, Riedel-Kruse I H, Nawroth J C, Roukes M L, Laurent G and Masmanidis S C 2009 High-resolution three-dimensional extracellular recording of neuronal activity with microfabricated electrode arrays J. Neurophysiol. 1011671

Elston G N 2003 Cortex, cognition and the cell: new insights into the pyramidal neuron and prefrontal function Cereb. Cortex 13 1124-38

Ferroli P, Franzini A, Marras C, Maccagnano E, Incerti L and Broggi G 2004 Simple method to assess accuracy of deep brain stimulation electrode placement: pre-operative stereotactic CT + postoperative MR image fusion Stereotact. Funct. Neurosurg. 82 14-9

Franceschetti S, Sancini G, Panzica F and Radici C 1998 Postnatal differentiation of firing properties and morphological characteristics in layer $\mathrm{V}$ pyramidal neurons of the sensorimotor cortex Neuroscience $\mathbf{8 3} 1013$

Freeman W J 2004 Origin, structure, and role of background EEG activity: part 2. Analytic phase Clin. Neurophysiol. 1152089

Freeman W J 2005 Origin, structure, and role of background EEG activity: part 3. Neural frame classification Clin. Neurophysiol. 1161118

Freeman W J 2006 Origin, structure, and role of background EEG activity: part 4. Neural frame simulation Clin. Neurophysiol. $117572-89$

Friel K et al 2007 Effects of a rostral motor cortex lesion on primary motor cortex hand representation topography in primates Neurorehabil. Neural Repair 21 51-61

Gabor D 1946 Theory of communication Electr. Eng. 93 429-57

Gage G J, Ludwig K A, Otto K J, Ionides E L and Kipke D R 2005 Naive coadaptive cortical control J. Neural Eng. 2 52-63

Gorman A L F 1966 Differential patterns of activation of pyramidal system elicited by surface anodal and cathodal cortical stimulation J. Neurophysiol. 29 547-64

Gradinaru V, Mogri M, Thompson K R, Henderson J M and Deisseroth K 2009 Optical deconstruction of parkinsonian neural circuitry Science 324 354-9

Hern J E, Landgren S, Phillips C G and Proter R 1962 Selective excitation of corticofugal neurones by surface-anodal stimulation of the baboon's motor cortex J. Physiol. 161 73-90

Humphrey D R, Schmidt E M and Thompson W D 1970 Predicting measures of motor performance from multiple cortical spike trains Science $\mathbf{1 7 0} 758$

Jando G, Carpi D, Kandel A and Urioste R 1995 Spike-and-wave epilepsy in rats: sex differences and inheritance of physiological traits Neuroscience 64301

Jensen K F and Killackey H P 1987 Terminal arbors of axons projecting to the somatosensory cortex of the adult rat. The normal morphology of specific thalamocortical afferents J. Neurosci. 7 3529-43

Kandel A and Buzsaki G 1997 Cellular-synaptic generation of sleep spindles, spike-and-wave discharges, and evoked thalamocortical responses in the neocortex of the rat J. Neurosci. 17 6783-97

Kipke D R, Vetter R J, Williams J C and Hetke J F 2003 Silicon-substrate intracortical microelectrode arrays for long-term recording of neuronal spike activity in cerebral cortex IEEE Trans. Neural Syst. Rehabil. Eng. 11151

Landau W M, Bishop G H and Clare M H 1964 Analysis of form + distribution of evoked cortical potentials under influence of polarizing currents J. Neurophysiol. 27 788-813

Larson P S, Richardson R M, Starr P A and Martin A J 2008 Magnetic resonance imaging of implanted deep brain stimulators: experience in a large series Stereotact. Funct. Neurosurg. 86 92-100 
Le Van Quyen M, Foucher J, Lachaux J P, Rodriguez E, Lutz A, Martinerie J and Varela F J 2001 Comparison of Hilbert transform and wavelet methods for the analysis of neuronal synchrony J. Neurosci. Methods 11183

Levine S P, Huggins J E, BeMent S L, Kushwaha R K, Schuh L A, Rohde M M, Passaro E A, Ross D A, Elisevich K V and Smith B J 2000 A direct brain interface based on event-related potentials IEEE Trans. Rehabil. Eng. 8 180-5

Libet B and Gerard R W 1941 Steady potential fields and neurone activity J. Neurophysiol. 4 438-55

Lund J S, Yoshioka T and Levitt J B 1993 Comparison of intrinsic connectivity in different areas of macaque monkey cerebral cortex Cereb. Cortex 3 148-62

Manger P R, Woods T M, Munoz A and Jones E G 1997 Hand/face border as a limiting boundary in the body representation in monkey somatosensory cortex J. Neurosci. 17 $6338-51$

Manola L, Holsheimer J, Veltink P and Buitenweg J R 2007 Anodal versus cathodal stimulation of motor cortex: a modeling study Clin. Neurophysiol. 118464

Marzullo T C, Miller C R and Kipke D R 2006 Suitability of the cingulate cortex for neural control IEEE Trans. Neural Syst. Rehabil. Eng. 14401

Matthews G 1977 Neural substrate for brain-stimulation reward in rat — cathodal and anodal strength-duration properties J. Comp. Physiol. Physiol. 91 858-74

McIntyre C C and Grill W M 1999 Excitation of central nervous system neurons by nonuniform electric fields Biophys. $J$. 76878

Mercanzini A, Cheung K, Buhl D L, Boers M, Maillard A, Colin P, Bensadoun J C, Bertsch A and Renaud P 2008 Demonstration of cortical recording using novel flexible polymer neural probes Sensors Actuators A 14390

Musallam S, Corneil B D, Greger B, Scherberger H and Andersen R A 2004 Cognitive control signals for neural prosthetics Science $\mathbf{3 0 5} 258-62$

Nitsche M A and Paulus W 2000 Excitability changes induced in the human motor cortex by weak transcranial direct current stimulation J. Physiol. 527 633-40

Nowak L G and Bullier J 1998a Axons, but not cell bodies, are activated by electrical stimulation in cortical gray matter: I. Evidence from chronaxie measurements Exp. Brain Res. 118477

Nowak L G and Bullier J 1998b Axons, but not cell bodies, are activated by electrical stimulation in cortical gray matter: II. Evidence from selective inactivation of cell bodies and axon initial segments Exp. Brain Res. 118489

Parikh H, Marzullo T C and Kipke D R 2009 Lower layers in the motor cortex are more effective targets for penetrating microelectrodes in cortical prostheses J. Neural Eng. 6026004

Picard R and Cook D 1984 Cross-validation of regression models J. Am. Statist. Assoc. 79 575-83
Plautz E J, Barbay S, Frost S B, Friel K M, Dancause N, Zoubina E V, Stowe A M, Quaney B M and Nudo R J 2003 Post-infarct cortical plasticity and behavioral recovery using concurrent cortical stimulation and rehabilitative training: a feasibility study in primates Neurol. Res. 25 801-10

Rosenblum M, Pikovsky A, Schafer C, Tass P A and Kurths J 2001 Phase synchronization: from theory to data analysis Handbook Biol. Phys. 4 279-321

Schwartz A B, Cui X T, Weber D J and Moran D W 2006 Brain-controlled interfaces: movement restoration with neural prosthetics Neuron 52 205-20

Serruya M D, Hatsopoulos N G, Paninski L, Fellows M R and Donoghue J P 2002 Brain-machine interface: instant neural control of a movement signal Nature 416141

Shaw J C 1996 The electroencephalogram: its patterns and origin. By John S Barlow J. Psychophysiol. 10174

Skoglund T, Pascher R and Berthold C 1997 The existence of a layer IV in the rat motor cortex Cereb. Cortex 7 178-80

Taub E, Uswatte G and Elbert T 2002 New treatments in neurorehabilitation founded on basic research Nat. Rev. Neurosci. $3228-36$

Taylor D M, Helms Tillery S I and Schwartz A B 2003 Information conveyed through brain-control: cursor versus robot IEEE Trans. Neural Syst. Rehabil. Eng. 11195

Teskey G C, Flynn C, Goertzen C D, Monfils M H and Young N A 2003 Cortical stimulation improves skilled forelimb use following a focal ischemic infarct in the rat Neurol. Res. 25794

Townsend G, Peloquin P, Kloosterman F, Hetke J F and Leung L S 2002 Recording and marking with silicon multichannel electrodes Brain Res. Protoc. 9 122-9

Vetter R J, Williams J C, Hetke J F, Nunamaker E A and Kipke D R 2004 Chronic neural recording using silicon-substrate microelectrode arrays implanted in cerebral cortex IEEE Trans. Biomed. Eng. 51 896-904

Wessberg J, Stambaugh C R, Kralik J D, Beck P D, Laubach M, Chapin J K, Kim J, Biggs S J, Srinivasan M A and Nicolelis M A L 2000 Real-time prediction of hand trajectory by ensembles of cortical neurons in primates Nature $\mathbf{4 0 8} 361$

Wongsarnpigoon A and Grill W M 2008 Computational modeling of epidural cortical stimulation J. Neural Eng. 5 443-54

Yazdan-Shahmorad A, Gage G J, Marzullo T C, Kim E and Kipke D R 2007 Linear electrode depth estimation in rat motor cortex by laminar analysis of ketamine-xylazine-induced oscillations $3 \mathrm{rd}$ Int. IEEE/EMBS Conf. on Neural Engineering vol 1 pp 646-9

Yazdan-Shahmorad A, Kipke D R and Lehmkuhle M J 2011 Polarity of cortical electrical stimulation differentially affects neuronal activity of deep and superficial layers of rat motor cortex Brain Stimulation at press

Yeomans J S, Maidment N T and Bunney B S 1988 Excitability properties of medial forelimb-bundle axons A10 Dopamine cells Brain Res. 450 86-93 